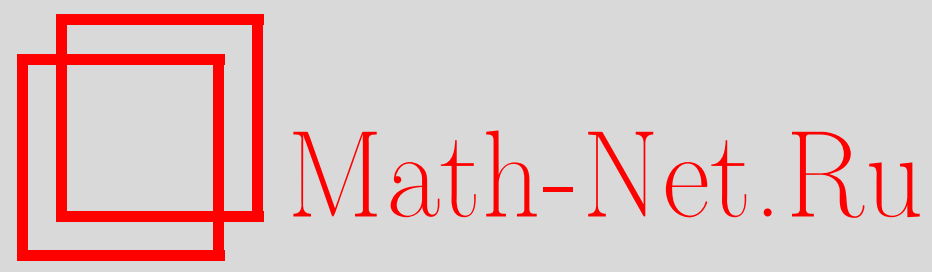

Р. О. Гринив, Я. В. Микитюк, О подобии возмущенных операторов умножения, Матем. заметки, 2001, том 70, выпуск $1,38-45$

DOI: https://doi.org/10.4213/mzm716

Использование Общероссийского математического портала Math-Net.Ru подразумевает, что вы прочитали и согласны с пользовательским соглашением http://www . mathnet.ru/rus/agreement

Параметры загрузки:

IP : 54.198 .187 .58

26 апреля 2023 г., $13: 27: 44$

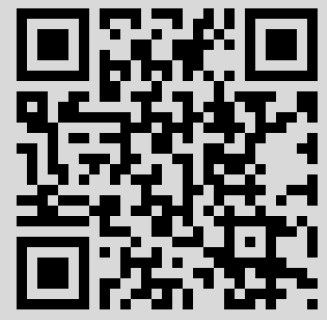




\title{
О ПОДОБИИ ВОЗМУЩЕННЫХ ОПЕРАТОРОВ УМНОЖЕНИЯ
}

\author{
Р. О. Гринив, Я.В. Микитюк
}

Пусть $S$ есть оператор умножения на независимую переменную $x$ в $L_{2}(0,1)$, а $V$ интегральный оператор вольтеррова типа. В заметке находятся достаточные условия для подобия операторов $T:=S+V$ и $S$ и обсуждаются некоторые обобщения полученных результатов в абстрактной ситуации.

Библиографоия: 12 названий.

1. Введение. Рассмотрим в гильбертовом пространстве $\mathscr{H}=L_{2}(0,1)$ оператор $S$ умножения на независимую переменную, $(S f)(x)=x f(x)$, и его возмущение $T:=S+V$, где

$$
(V f)(x):=\int_{0}^{x} v(x, t) f(t) d t .
$$

На протяжении всей работы предполагается, что ядро $v$ измеримо по Лебегу на $[0,1] \times$ $[0,1], v(x, t)=0$ при $x<t$ и что интегральный оператор $V$ ограничен в $\mathscr{H}$.

Операторы такого вида возникают, например, в модели Фридрихса [1], [2] или в химии полимеров, где $T$ описывает эволюцию системы полимеров вблизи динамического равновесия [3]. В обоих примерах существенньй интерес составляет вопрос об асимптотическом поведении групшы $e^{i t T}$ (в частности, ее равномерная ограниченность, или устойчивость по Ляпунову), что порождает задачу о подобии оператора $T$ самосопряженному. Для случая, когда $v(x, t)=\varphi(x) \psi(t)$ при $0 \leqslant t \leqslant x \leqslant 1$ и $\varphi, \psi \in \mathscr{H}$, эта задача детально исследовалась в статье [4], где установлен следующий результат.

Теорема А. Пусть $\varphi \psi \equiv 0$ и существуют модули непрерывности $\omega_{1}, \omega_{2}$ такие, что $\varphi \in \operatorname{Lip}\left(\omega_{1}\right), \psi \in \operatorname{Lip}\left(\omega_{2}\right) u$

$$
\int_{0} \frac{\omega_{1}(\tau) \omega_{2}(\tau)}{\tau}<\infty
$$

Тогда оператор Т подобен самосопряженному.

Более того, на основании тонкого анализа поведения резольвенты оператора $T$ вблизи вещественной оси показана точность условия (2) в том смысле, что при его нарушении оператор $T$ уже не обязан быть подобным самосопряженному; в [4] построены соответствуюшие примеры для $\omega_{1}(\tau)=|\ln \tau|^{-\delta_{1}}$ и $\omega_{2}(\tau)=|\ln \tau|^{-\delta_{2}}$ с $\delta_{1}+\delta_{2}<1$.

Основная цель настоящей статьи - найти условия, при которых возмущенньй оператор $T$ подобен невозмущенному оператору $S$. Мы рассматриваем возмущения $V$ общего 
вида (1) и применяем метод, берущий начало от Фридрихса [1]. А именно, мы находим достаточные условия для существования ограниченного оператора $K$ со спектральным радиусом меньше 1, удовлетворяющего соотношению

$$
T(I+K)=(I+K) S
$$

Основные результаты работы следующие.

ТЕОРема 1. Предположим, что ядро

$$
w(x, t):=\frac{|v(x, t)|}{x-t}, \quad x, t \in[0,1]
$$

порождает ограниченный интегральный оператор со спектральным радиусом $\mathrm{r}(W)$ меньше $1 / 2$. Тогда операторы $T$ и $S$ подобны.

СлЕДСТвИЕ 1. Пусть существует функиия $q \in L_{1}(0,1)$ такая, что $w(x, t) \leqslant$ $q(x-t)$ для всех $x, t, 0 \leqslant t<x \leqslant 1$. Тогда операторы $T$ и $S$ подобны.

Заметим, что в предположениях теоремы А вьполняется оценка

$$
\begin{aligned}
|v(x, t)| & \leqslant|\varphi(x) \psi(t)|+|\varphi(t) \psi(x)|=(|\varphi(x)|-|\varphi(t)|)(|\psi(t)|-|\psi(x)|) \\
& \leqslant \omega_{1}(x-t) \omega_{2}(x-t)
\end{aligned}
$$

поэтому следствие 1 применимо с $q(\tau)=\omega_{1}(\tau) \omega_{2}(\tau) / \tau$, что доказывает даже более сильное утверждение о подобии $T$ невозмушенному оператору $S$. Следствие 1 также применимо к ядрам $v$ с конечной нормой

$$
\|v\|_{\alpha}:=\operatorname{ess~sup}_{0 \leqslant t<x \leqslant 1}(x-t)^{1-\alpha}|v(x, t)|
$$

при некотором $\alpha>0$ (см. [5], [6] и указанную там библиографию относительно подобия вольтерровых операторов операторам дробного интегрирования). Отметим также, что, как следует из [4], условие $q \in L_{1}(0,1)$ нельзя заменить более слабым.

На самом деле, при нашем подходе оператор $V$ даже не обязан быть интегральным вида (1). Допускаются операторы $V$, которые мажорируются в некотором смысле и принадлежат алгебре $\mathscr{A}$ операторов, оставляющих инвариантньм и подпространства функций с носителем в $[a, 1], a \in[0,1)$. Соответствующие понятия привлекают теорию неотрицательных операторов в банаховых пространствах с положительным конусом [7] и вводятся в п. 2. Абстрактные результаты о подобии операторов $T$ и $S$ устанавливаются в п. 3 и затем используются в п. 4 для доказательства теоремы 1 и следствия 1. В последнем пункте обсуждаются некоторые непосредственные обобщения полученных результатов.

На протяжении всей работы $\mathrm{r}(T)$ обозначает спектральный радиус ограниченного оператора $T$; напомним, что $r(T)=\lim _{n \rightarrow \infty}\left\|T^{n}\right\|^{1 / n}$. 
2. Неотрицательные операторы и вспомогательные результаты. Прежде всего, напомним некоторые понятия теории линейных пространств с положительным конусом (см., например, [7]). Обозначим через

$$
\mathscr{H}_{+}=\{f \in \mathscr{H} \mid f(x) \geqslant 0 \text { п.в. в }[0,1]\}
$$

и

$$
\mathscr{B}_{+}(\mathscr{H})=\left\{A \in \mathscr{B}(\mathscr{H}) \mid A \mathscr{H}_{+} \subset \mathscr{H}_{+}\right\}
$$

конусы неотрицательньх элементов пространства $\mathscr{H}$ и неотрицательных ограниченных операторов в $\mathscr{H}$ соответственно. Как обычно, для произвольных $f, g \in \mathscr{H}$ и $A, B \in \mathscr{B}(\mathscr{H})$ мы пишем $f \geqslant g$ и $A \geqslant B$, если $f-g \in \mathscr{H}_{+}$и $A-B \in \mathscr{B}_{+}(\mathscr{H})$ соответственно. Напомним, что конус $\mathscr{H}_{+}$является миниэдральным, и поэтому для любого элемента $f \in \mathscr{H}$ существует его модуль $|f|$ из $\mathscr{H}_{+}$; в данном контексте $|f|$ является функцией, определенной посредством равенства $|f|(x)=|f(x)|$. Напротив, конус $\mathscr{B}+(\mathscr{H})$ не является миниэдральным, и модуль $|A|$ нельзя определить для всех $A \in \mathscr{B}(\mathscr{H})$. Мы укажем класс операторов, модуль которых корректно определен.

Мы говорим, что оператор $B$ мажсорирует $A$ (и пишем $B \succ A$ или $A \prec B$ ), если $|A f| \leqslant B|f|$ для всех $f \in \mathscr{H}$. Если $A \prec B$, то, как легко видеть, $B$ неотрицателен и

$$
|(A f, g)| \leqslant(|A f|,|g|) \leqslant(B|f|,|g|) \leqslant\|B\|\|f\|\|g\|
$$

при всех $f, g \in \mathscr{H}$, т.е. $\|A\| \leqslant\|B\|$. Более того, если $B$ мажорирует $A$, то неравенства

$$
\left|A^{n} f\right| \leqslant B\left|A^{n-1} f\right| \leqslant \cdots \leqslant B^{n-1}|A f| \leqslant B^{n}|f| \quad \forall f \in \mathscr{H}
$$

показывают, что $A^{n} \prec B^{n}$ для всех $n \in \mathbb{N}$ и потому $\mathrm{r}(A) \leqslant \mathrm{r}(B)$.

Положим

$$
\mathscr{M}(A):=\left\{B \in \mathscr{B}_{+}(\mathscr{H}) \mid B \succ A\right\}
$$

И

$$
\mathscr{B}_{\mathscr{M}}(\mathscr{H}):=\{A \in \mathscr{B}(\mathscr{H}) \mid \mathscr{M}(A) \neq \varnothing\} .
$$

Заметим, что $\mathscr{B} \mathscr{M}(\mathscr{H})$ является мультипликативньп конусом в $\mathscr{B}(\mathscr{H})$, т.е. $A B \in$ $\mathscr{B} \mathscr{M}(\mathscr{H})$, как только $A, B \in \mathscr{B} \mathscr{M}(\mathscr{H})$.

ПримеР. Пусть $A$ и $B$ - интегральные операторы в $\mathscr{H}$ с непрерывными ядрами $a$ и $b$ соответственно. Тогда $B \in \mathscr{M}(A)$, если и только если $|a(x, t)| \leqslant b(x, t)$ везде в $[0,1] \times[0,1]$, a $A \in \mathscr{B} \mathscr{M}(\mathscr{H})$ тогда и только тогда, когда ядро $|a|$ порождает ограниченный интегральньй оператор в $\mathscr{H}$.

В некотором смысле предыдущий пример является общим, так как любой ограниченньй оператор в $\mathscr{H}$ есть сильньй предел интегральных операторов с непрерьвньми ядрами. В самом деле, пусть $r$ есть гладкая неотрицательная функция, удовлетворяюшая условиям supp $r \subset[0,1]$ и $\int r=1$. Обозначим через $R_{\varepsilon}$ интегральньй оператор

$$
\left(R_{\varepsilon} f\right)(x)=\int_{0}^{1} r_{\varepsilon}(x-t) f(t) d t, \quad r_{\varepsilon}(x)=\varepsilon^{-1} r\left(\frac{x}{\varepsilon}\right)
$$


и для любого $A \in \mathscr{B}(\mathscr{H})$ и $\varepsilon>0$ положим

$$
A_{\varepsilon}:=R_{\varepsilon} A R_{\varepsilon}
$$

Заметим, что $R_{\varepsilon} \rightarrow I$ при $\varepsilon \rightarrow 0$ сильно в $\mathscr{H}$ [8, гл. III.2], поэтому

$$
\mathrm{s}-\lim _{\varepsilon \rightarrow 0} A_{\varepsilon}=A .
$$

Более того, поскольку $R_{\varepsilon}$ является оператором Гильберта-Шмидта, то $A_{\varepsilon}$ есть интегральньй оператор с некоторым ядром $a_{\varepsilon}$ (см. подробности, например, в [9]). Обозначая через $\delta_{\xi}(u):=\delta(u-\xi)$ дельта-функцию Дирака в точке $\xi$, мы видим, что

$$
a_{\varepsilon}(x, t)=\left(A_{\varepsilon} \delta_{t}, \delta_{x}\right)=\left(A R_{\varepsilon} \delta_{t}, R_{\varepsilon}^{*} \delta_{x}\right)=\left(A r_{\varepsilon, t}, \hat{r}_{\varepsilon, x}\right)
$$

где $r_{\varepsilon, t}(u):=r_{\varepsilon}(u-t)$ и $\hat{r}_{\varepsilon, x}(u):=r_{\varepsilon}(x-u)$ являются непрерывными функциями по $t$ и $x$ соответственно со значениями в $\mathscr{H}$, и поэтому ядро $a_{\varepsilon}$ непрерьвно.

Это замечание используется существенным образом при доказательстве следующего утверждения.

Лемма 1. Для каждого $A \in \mathscr{B} \mathscr{M}(\mathscr{H})$ множество $\mathscr{M}(A)$ содержит минимальный әлемент $|A|$, называемый модулем $A$. Иными словами, $|A| \in \mathscr{M}(A) u|A| \leqslant B$ для всех $B \in \mathscr{M}(A)$.

ДокаЗАТЕЛЬСТво. Предположим, что $A \in \mathscr{B} \mathscr{M}(\mathscr{H})$ и $B \in \mathscr{M}(A)$ и положим $A_{\varepsilon}=$ $R_{\varepsilon} A R_{\varepsilon}, B_{\varepsilon}:=R_{\varepsilon} B R_{\varepsilon}$, где интегральньй оператор $R_{\varepsilon}$ построен вьше. Тогда

$$
\begin{aligned}
\left|\left(A_{\varepsilon} f, g\right)\right| & =\left|\left(A R_{\varepsilon} f, R_{\varepsilon}^{*} g\right)\right| \leqslant\left(B\left|R_{\varepsilon} f\right|,\left|R_{\varepsilon}^{*} g\right|\right) \\
& \leqslant\left(B R_{\varepsilon}|f|, R_{\varepsilon}^{*}|g|\right)=\left(B_{\varepsilon}|f|,|g|\right)
\end{aligned}
$$

для всех $f, g \in \mathscr{H}$, что влечет неравенство

$$
\left|a_{\varepsilon}(x, t)\right| \leqslant b_{\varepsilon}(x, t)
$$

для соответствующих ядер.

Обозначим через $A_{\varepsilon}^{+}$интегральньй оператор, порожденньй ядром $\left|a_{\varepsilon}(x, t)\right|$. Ввиду неравенства (4) оператор $A_{\varepsilon}^{+}$ограничен, $A_{\varepsilon}^{+} \leqslant B_{\varepsilon}$ и $\left\|A_{\varepsilon}^{+}\right\| \leqslant\left\|B_{\varepsilon}\right\|$. Более того, по построению $A_{\varepsilon}^{+} \succ A_{\varepsilon}$ при всех $\varepsilon>0$. Поскольку $B_{\varepsilon} \rightarrow B$ при $\varepsilon \rightarrow 0$ сильно, нормы $\left\|A_{\varepsilon}^{+}\right\|$ равномерно ограничены по $\varepsilon>0$ и множество $\left\{A_{\varepsilon}^{+}\right\}_{\varepsilon>0}$ слабо компактно в $\mathscr{B}(\mathscr{H})$. Поэтому существуют последовательность $\varepsilon_{n} \rightarrow 0$ и оператор $A_{0}^{+}$такие, что $A_{\varepsilon_{n}}^{+} \rightarrow A_{0}^{+}$при $n \rightarrow \infty$ слабо. Переходя к пределу при $\varepsilon_{n} \rightarrow 0$ в неравенствах $A_{\varepsilon}^{+} \leqslant B_{\varepsilon}$ и $A_{\varepsilon}^{+} \succ A_{\varepsilon}$, мы заключаем, что $A_{0}^{+} \leqslant B$ и $A_{0}^{+} \succ A$. Так как $B \in \mathscr{M}(A)$ был произвольным, последние соотношения доказьвают, что $A_{0}^{+}$является слабьм пределом $A_{\varepsilon}^{+}$при $\varepsilon \rightarrow 0$ и что $A_{0}^{+}$и есть искомьй модуль $|A|$ оператора $A$.

Пусть $\chi_{a}$ обозначает оператор умножения на индикатор интервала $[a, 1]$ и

$$
\mathscr{A}:=\left\{A \in \mathscr{B}(\mathscr{H}) \mid A \chi_{a}=\chi_{a} A \chi_{a} \quad \forall a \in[0,1]\right\} .
$$

Ясно, что $\mathscr{A}$ есть слабо замкнутая подалгебра $\mathscr{B}(\mathscr{H})$. Положим

$$
\mathscr{A}_{+}:=\mathscr{A} \cap \mathscr{B}_{+}(\mathscr{H})
$$

и заметим, что $|A| \in \mathscr{A}_{+}$для всех $A \in \mathscr{B} \mathscr{M}(\mathscr{H}) \cap \mathscr{A}$. 
Замечание. Предположим, что $A \in \mathscr{A}_{+}$и $A_{\varepsilon}=R_{\varepsilon} A R_{\varepsilon}, \varepsilon>0$. Тогда $A_{\varepsilon}$ есть интегральньй оператор с непрерывным ядром $a_{\varepsilon}(x, t)$, причем $a_{\varepsilon}(x, t)=0$ при $t>x$. Поэтому $A_{\varepsilon}$ является вольтерровым оператором (см., например, $[10$, п. 68]) и $A$ является сильным пределом вольтерровых операторов.

Это замечание влечет следующий результат.

Лемма 2. Пусть $A \in \mathscr{A}_{+} ;$тогда $[S, A]:=S A-A S$ принадлежит $\mathscr{A}_{+}$.

ДокаЗАтЕльство. Достаточно заметить, что $\left[S, A_{\varepsilon}\right]$ является интегральным оператором с неотрицательным ядром $(x-t) a_{\varepsilon}(x, t)$ и поэтому принадлежит $\mathscr{A}_{+}$.

3. Подобие операторов $S$ и $T$. В этом пункте мы изучим вопрос о существовании оператора $K \in \mathscr{B}(\mathscr{H})$, удовлетворяющего уравнению (3). Полагая $[S, K]:=S K-K S$, запишем (3) в виде уравнения

$$
[S, K]+V K+V=0
$$

и решим последнее методом последовательных приближений, восходяшим к Фридрихсу $[1$, гл. II.6].

Лемма 3. Предположим, ито $V \in \mathscr{B} \mathscr{M}(\mathscr{H}) \cap \mathscr{A} u[S, W] \in \mathscr{M}(V)$ для некоторого оператора $W \in \mathscr{A}_{+}$. Тогда уравнение

$$
[S, K]=V
$$

имеет решение $K \in \mathscr{B} \mathscr{M}(\mathscr{H}) \cap \mathscr{A}$ такое, что $K \prec W$.

ДокАЗАТЕЛЬСТво. Поскольку $[S, W] \succ V$, при всех $\varepsilon>0$ выполнено неравенство $R_{\varepsilon}[S, W] R_{\varepsilon} \succ R_{\varepsilon} V R_{\varepsilon}=: V_{\varepsilon}$. Ввиду леммы $2 R_{\varepsilon} S \leqslant S R_{\varepsilon}$, а следовательно,

$$
R_{\varepsilon}[S, W] R_{\varepsilon}=R_{\varepsilon} S W R_{\varepsilon}-R_{\varepsilon} W S R_{\varepsilon} \leqslant S W_{\varepsilon}-W_{\varepsilon} S=\left[S, W_{\varepsilon}\right]
$$

и $\left[S, W_{\varepsilon}\right] \succ V_{\varepsilon}$, где $W_{\varepsilon}=R_{\varepsilon} W R_{\varepsilon}$. Отсюда следует, что ядра $v_{\varepsilon}$ и $w_{\varepsilon}$ операторов $V_{\varepsilon}$ и $W_{\varepsilon}$ удовлетворяют неравенству

$$
\left|\frac{v_{\varepsilon}(x, t)}{x-t}\right| \leqslant w_{\varepsilon}(x, t)
$$

в частности, $V_{\varepsilon} \in \mathscr{A}$. Обозначим через $K^{(\varepsilon)}$ интегральный оператор, порожденньй ядром $v_{\varepsilon}(x, t) /(x-t)$. Ввиду последнего неравенства $K^{(\varepsilon)}$ является ограниченным оператором, принадлежащим алгебре $\mathscr{A}, K^{(\varepsilon)} \prec W_{\varepsilon}$ и $\left[S, K^{(\varepsilon)}\right]=V_{\varepsilon}$. Поэтому нормы $\left\|K^{(\varepsilon)}\right\|$ равномерно ограничены по $\varepsilon>0$ и существует ограниченный оператор $K$ и последовательность $\varepsilon_{n} \rightarrow 0$ такая, что при $n \rightarrow \infty K^{\left(\varepsilon_{n}\right)}$ слабо сходятся к $K$. Переходя к пределу при $\varepsilon_{n} \rightarrow 0$ в соотношениях

$$
S K^{(\varepsilon)}-K^{(\varepsilon)} S=V_{\varepsilon}, \quad K^{(\varepsilon)} \prec W_{\varepsilon}
$$

мы убеждаемся в том, что $K$ есть решение уравнения $[S, K]=V$ и удовлетворяет неравенству $K \prec W$. Лемма доказана. 
СледСтвие 2. Предположим, что $U, V \in \mathscr{B} \mathscr{M}(\mathscr{H}) \cap \mathscr{A} u[S, W] \in \mathscr{M}(V)$ для некоторого $W \in \mathscr{A}_{+}$. Тогда уравнение

$$
[S, K]=V U
$$

обладает решением $K \in \mathscr{B} \mathscr{M}(\mathscr{H}) \cap \mathscr{A}$ таким, что $K \prec W|U|$.

ДокаЗАтЕльСтво. Поскольку $[S, W|U|]=[S, W]|U|+W[S,|U|] \geqslant[S, W]|U| \succ V U$ благодаря лемме 2 и предположениям следствия, лемма 3 применима с $W|U|$ и $V U$ вместо $W$ и $V$ и утверждение доказано.

Tеорема 2. Предположим, ито $V \in \mathscr{A}$ и что существует оператор $W \in \mathscr{A}_{+}$ такой, что $\mathrm{r}(W)<1 u[S, W] \succ V$. Тогда уравнение

$$
[S, K]+V K+V=0
$$

имеет решение $K \in \mathscr{B} \mathscr{M}(\mathscr{H}) \cap \mathscr{A}$.

ДоКАЗАТЕЛЬСТво. Будем искать $K$ в виде

$$
K=\sum_{k=1}^{\infty} K_{n}
$$

где $K_{n}$ находятся рекуррентно из соотношений

$$
\left[S, K_{n}\right]=-V K_{n-1}, \quad n \in \mathbb{N},
$$

а $K_{0}:=I$. Используя лемму 3 и следствие 2 , мы находим последовательно $K_{n} \in \mathscr{A}$, $n=1,2, \ldots$, такие, что $K_{n} \prec W\left|K_{n-1}\right| \prec W^{n}$. Поэтому $\left\|K_{n}\right\| \leqslant\left\|W^{n}\right\|$ и ряд $\sum_{n=1}^{\infty} K_{n}$ сходится абсолютно в равномерной операторной топологии, а его сумма $K$ удовлетворяет равенству

$$
[S, K]=\sum_{n=1}^{\infty}\left[S, K_{n}\right]=-\sum_{n=1}^{\infty} V K_{n-1}=-V K-V .
$$

Теорема доказана.

СлЕдСтвиЕ 3. Если в предположсениях теоремы $2 \mathrm{r}(W)<1 / 2$, то уравнение (5) имеет решение $K \in \mathscr{B} \mathscr{M}(\mathscr{H}) \cap \mathscr{A} c \mathrm{r}(K)<1$.

ДокаЗАТЕльСтво. Заметим, что решение $K$, построенное при доказательстве теоремы 2 , удовлетворяет неравенству $K \prec W(I-W)^{-1}$. Поэтому $\mathrm{r}(K) \leqslant \mathrm{r}\left(W(I-W)^{-1}\right)$ и остается отметить, что $\mathrm{r}\left(W(I-W)^{-1}\right)<1$ при $\mathrm{r}(W)<1 / 2$.

\section{4. Доказательство основных результатов.}

ДокАЗАТЕЛЬСТво ТЕОРЕмЫ 1. Для доказательства теоремы достаточно заметить, что предположения теоремы 2 и следствия 3 удовлетворены для интегрального оператора $W$ с ядром

$$
w(x, t):=\frac{|v(x, t)|}{x-t} .
$$


ТЕорема 3. Пусть ш есть полохительное ядро на $[0,1] \times[0,1]$ такое, что $w(x, t)=0$ при $t>x u w(x, t) \leqslant q(x-t) n p u x \geqslant t$, где $q \in L_{1}(0,1)$. Тогда порохсдаемый интегральный оператор $W$ является вольтерровым в $\mathscr{H}$ (т.е. $W$ компактен $u \mathrm{r}(W)=0) u\|W\| \leqslant\|q\|_{1}$.

ДоказАТЕЛьство. Докажем сначала, что $W$ ограничен в $\mathscr{H}$. В самом деле,

$$
\begin{aligned}
& \int_{0}^{x} w(x, t) d t \leqslant \int_{0}^{x} q(x-t) d t=\int_{0}^{x} q(t) d t \leqslant\|q\|_{1}, \\
& \int_{t}^{1} w(x, t) d x \leqslant \int_{t}^{1} q(x-t) d x=\int_{0}^{1-t} q(x) d x \leqslant\|q\|_{1},
\end{aligned}
$$

поэтому $\|W\| \leqslant\|q\|_{1}$ благодаря тесту Шура [9, теорема 5.2]. Далее, положим

$$
w^{(m)}= \begin{cases}w(x, t), & \text { если } q(x-t) \leqslant m, \\ 0, & \text { если } q(x-t)>m .\end{cases}
$$

Тогда порождаемый интегральньй оператор $W^{(m)}$ является вольтерровым в $\mathscr{H}$ (см., например, [10, п. 68]) и

$$
\left\|W-W^{(m)}\right\| \leqslant \int_{q>m} q(t) d t \rightarrow 0
$$

при $m \rightarrow \infty$ ввиду вышеприведенных рассуждений и того, что $q \in L_{1}(0,1)$. Следовательно, $W$ также вольтерров и теорема доказана.

Следствие 1 теперь легко вьводится из теорем 1 и 3.

5. Некоторые обобщения. В этом пункте мы обсудим некоторые непосредственные обобщения основных результатов. Заметим сначала, что рассуждения пунктов 2 и 3 остаются справедливыми для банаховых пространств $L_{p}(a, b)$ с произвольными $-\infty \leqslant$ $a<b \leqslant \infty$ и $p \in[1, \infty)$. Далее, можно заменить $S$ оператором умножения на произвольную возрастающую функцию $\varphi(x)$. Аналог теоремы 1 и следствия 1 формулируется следующим образом.

ТЕОРема 1'. Предположим, что ч строго возрастает на $(a, b)$ и что ядро

$$
w(x, t):=\left|\frac{v(x, t)}{\varphi(x)-\varphi(t)}\right|
$$

порохдает ограниченный интегральный оператор в $L_{p}(a, b)$ со спектральным радиусом меньше 1/2. Тогда операторы $S$ умножения на $\varphi$ и $T:=S+V$, где

$$
(V f)(x):=\int_{a}^{x} v(x, t) f(t) d e,
$$

подобны в $L_{p}(a, b)$. В частности, $S$ и $T$ подобнь, если $w(x, t) \leqslant q(x-t)$ для некоторой $q \in L_{1}(a, b)$.

Отметим также, что большинство результатов остаются в силе для произвольной банаховой решетки $\mathscr{X}$ функций на $(a, b)[11]$ при условии, что тождественньй оператор в $\mathscr{X}$ есть сильньй предел интегральных операторов с непрерьвными ядрами.

ЗАмЕчАниЕ. После того как настоящая статья была завершена, М. М. Маламуд обратил наше внимание на его заметку [12], где были анонсированы без доказательства утверждения теоремы 1 в предположении $\mathrm{r}(W)=0$ и следствия 1 . 


\section{СПИСОК ЦИТИРОВАННОЙ ЛИТЕРАТУРЫ}

[1] Фридрихс К. Возмущение спектра операторов в гильбертовом пространстве. М.: Мир, 1969.

[2] Фаддеев Л. Д. О модели Фридрихса в теории возмущения непрерьвного спектра // Тр. МИАН. 1964. Т. 73. С. 292-313.

[3] Kokholm N.J. Spectral analysis of perturbed multiplication operators occurring in polymerisation chemistry // Proc. Roy. Soc. Edinburgh. Sect. A. 1989. V. 113. № 1-2. P. 119-148.

[4] Naboko S. N., Tretter C. Lyapunov stability of a perturbed multiplication operator // Contributions to Operator Theory in Spaces with an Indefinite Metric. The Heinz Langer Anniversary Volume on the Occasion of his 60th Birthday. Oper. Theory Adv. Appl. V. 106. Basel: Birkhäuser, 1998. P. 309-326.

[5] Freeman J. M. Volterra operators similar to $J: f(x) \mapsto \int_{0}^{x} f(y) d y / /$ Trans. Amer. Math. Soc. 1965. V. 116. P. 181-192.

[6] Маламуд М. М. Подобие операторов Вольтерра и связанные вопросы теории дифференциальных уравнений дробного порядка // Тр. ММО. 1994. Т. 55. С. 73-148.

[7] Крейн М.Г., Рутман М. А. Линейные операторы, оставляющие инвариантным конус в пространстве Банаха // УМН. 1948. Т. 3. №1(23). С. 3-95.

[8] Стейн И. Сингулярные интегралы и дифференциальные свойства функций. М.: Мир, 1973.

[9] Халмош П., Сандер В. Ограниченные интегральные операторы в пространствах $L^{2}$. M.: Наука, 1985.

[10] Рисс Ф., Секефальви-Надь Б. Лекции по функциональному анализу. М.: Мир, 1979.

[11] Schaefer H. H. Banach Lattices and Positive Operators. Berlin: Springer, 1974.

[12] Маламуд М. М. О приведении операторов с непрерывным спектром к диагональному виду // УМH. 1977. Т. 32. № 5. С. 167.

(Р. О. Гринив) Институт прикладных проблем механики и математики

(Я.В. Микитюк) Львовский национальый университет им. И. Франко

E-mail: hryniv@mebm.lviv.ua, yamykytyuk@yahoo.com 